\title{
Transfusão Intra-uterina na Isoimunização Materna pelo Fator Rh
}

\author{
Intrauterine Transfusion in Maternal Rh Immunization
}

\begin{abstract}
Antonio Carlos Vieira Cabral, Marcos Roberto Taveira, Ana Paula Brum Miranda Lopes
Alamanda Kfoury Pereira, Henrique Vitor Leite
\end{abstract}

\section{RESUMO}

\begin{abstract}
Objetivos: avaliar os resultados do tratamento intra-útero de fetos anêmicos devido a isoimunização materna pelo fator $R h$.

Pacientes e Métodos: foram acompanhados 61 fetos submetidos a transfusão intra-uterina seja por via intraperitoneal, intravascular ou combinada. Os casos de fetos hidrópicos corresponderam a 19,7\% do total, sendo que nestes a via de tratamento sempre foi a intravascular. Foram realizadas em média 2,7 transfusões por feto, com um total de 163 procedimentos. A indicação para a transfusão se baseou na espectrofotometria do líquido amniótico (método de Liley) ou quando a dosagem de hemoglobina em sangue de cordão obtido por cordocentese fosse menor que $10 \mathrm{~g} / \mathrm{dL}$.

Resultados: em metade dos casos submetidos a transfusão intra-uterina, empregou-se a via intravascular. Em relação aos casos de fetos hidrópicos a sobrevida foi de $46 \%$ e nos fetos não-hidrópicos, de 84\%. Não ocorreram complicações maternas relacionadas ao procedimento. A idade média da interrupção da gestação foi de 34,8 semanas.

Conclusões: apesar da melhora do resultado com a transfusão intra-uterina guiada pelo ultra-som e da cordocentese, $a$ isoimunização materna pelo fator Rh permanece como causa de elevada morbimortalidade perinatal.
\end{abstract}

PALAVRAS-CHAVE: Isoimunização Rh. Transfusão intra-uterina. Terapia fetal.

\section{Introdução}

Embora a imunoprofilaxia com a utilização da imunoglobulina anti-Rh tenha reduzido em muito o surgimento de novos casos de imunização materna, o problema ainda existe, tanto em países subdesenvolvidos quanto em grandes centros de atendimento em países desenvolvidos ${ }^{1}$.

A anemia fetal secundária a hemólise devida à ação dos anticorpos maternos anti-Rh é reconhecida há mais de seis décadas, sendo responsável por elevada morbimortalidade perinatal quando não abordada em tempo hábil, tanto do ponto de vista do diagnóstico quanto do tratamento indicado ${ }^{2}$.

O diagnóstico do grau de anemia do concepto ainda só pode ser estabelecido de maneira invasiva, o que pode acarretar sérios riscos para a gestante e o concepto. Pode ser realizado de forma direta por meio da determinação dos níveis

Centro de Medicina Fetal do Hospital das Clínicas da Universidade Federal de Minas Gerais.

Correspondência: Antônio Carlos Vieira Cabral

Rua dos Otoni 909 sala 1804

30150-270 - Belo Horizonte - MG hematimétricos no sangue fetal coletado pela cordocentese ou de forma indireta pelo estudo espectrofotométrico do líquido amniótico obtido por meio de amniocentese, em que se determina o nivel de bilirrubina levando-se em consideração a idade gestacional. O resultado obtido no líquido amniótico deve ser comparado com algumas das curvas-padrão descritas, sendo que a mais utilizada é a de Liley, publicada em $1961^{3-5}$.

O ideal seria o emprego de métodos nãoinvasivos para a determinação do grau de anemia. Porém, nenhum método não-invasivo proporcionou segurança para estabelecer o real nivel da anemia fetal visando a indicação de terapia invasiva. São exemplos destes métodos a cardiotocografia ${ }^{6}$ e a dopplerfluxometria arterial e venosa ${ }^{7}$.

O tratamento intra-uterino para correção da anemia fetal por meio da transfusão sangüínea começou a ser realizado há mais de trinta anos, pioneiramente por Liley ${ }^{4,8}$. Ao longo dos últimos anos a correção da anemia fetal passou a ser mais efetiva e segura em decorrência do desenvolvimento da ultra-sonografia e de procedimentos intravasculares ${ }^{2}$.

A transfusão intra-uterina, que inicialmen- 
te era realizada exclusivamente pela via intraperitoneal, apresentou modificações técnicas que passaram pela transfusão na veia umbilical intra-hepática ${ }^{9}$, transfusão nos vasos do cordão umbilical $^{10}$ e ex-sangüíneo transfusão fetal por meio da cordocentese ${ }^{11}$.

Os resultados da transfusão intra-uterina são díspares ao longo das últimas três décadas e divergentes conforme os diversos autores. Gregg e Hutchinson ${ }^{12}$ relatam sobrevida perinatal de $33 \%$ e Hardyment et al. ${ }^{13}$ obtiveram aumento na taxa de sobrevida, elevando-a para $48 \%$. Ambos os autores utilizavam exclusivamente as transfusões intraperitoneais para seus procedimentos. Após o início da técnica intravascular houve grande melhoria dos resultados perinatais, principalmente devido à melhora da sobrevida dos fetos hidrópicos, conforme assinalam Doyle et al. ${ }^{14}$ que relatam resultados perinatais favoráveis em $73 \%$ dos casos acompanhados. Em nosso meio, Vitorello et al. ${ }^{15}$ encontraram resultados semelhantes, mostrando a importância do tratamento intra-útero. Mais recentemente são relatadas taxas de sobrevida perinatal entre 80 e $85 \%$ no tratamento da anemia fetal ${ }^{15-17}$.

O Centro de Medicina Fetal do Hospital das Clínicas da UFMG (Belo Horizonte) é referência regional no tratamento do feto anêmico e com o objetivo de comparar os resultados obtidos em nosso meio com aqueles observados em outros serviços, realizamos a presente avaliação dos nossos resultados.

\section{Pacientes e Métodos}

No período de janeiro de 1991 a julho de 1999 foram acompanhados 61 fetos de gestantes isoimunizadas em que houve a necessidade de tratamento intra-uterino para correção da anemia fetal. Foram realizadas 163 transfusões intrauterinas, com média de 2,7 procedimentos por paciente, sendo que o número de procedimentos variou em cada caso entre um e seis.

Os critérios para definição da anemia fetal foram determinados pelo estudo espectrofotométrico do líquido amniótico (curva de Liley) ${ }^{4}$ ou quando da realização da cordocentese encontrou-se uma hemoglobina inferior a $10 \mathrm{~g} / \mathrm{dL}$. A cordocentese não é utilizada de forma rotineira para a determinação do nivel de hemoglobina, devido ao risco do agravamento da doença pelo procedimento pela possibilidade de ocorrer macrotransfusão feto-materna.

As transfusões intra-uterinas sempre foram realizadas pelos mesmos profissionais, sendo que um orientava a punção e o outro, paramentado, realizava a punção do cordão umbilical, colhia o sangue para as dosagens e realizava a infusão do sangue, sendo a freqüência cardiaca fetal acompanhada durante todo o procedimento.

Utilizaram-se no período as técnicas de transfusão intraperitoneal e intravascular obedecendo-se a alguns critérios fundamentais, a saber: os fetos hidrópicos foram sempre submetidos à via intravascular e os não-hidrópicos à técnica de maior facilidade de acesso, sendo que em 4 casos utilizaram-se as duas vias. Nos casos de placenta anterior e fúndica fez-se a opção da via intravascular e naqueles de placenta posterior, a via intraperitoneal. A punção nos casos de transfusão intravascular foi sempre efetuada na veia umbilical. Não se utilizaram em nenhum caso bloqueadores neuromusculares, tocolíticos e antibioticoterapia profilática.

Em um caso de feto hidrópico ocorreu bradicardia e óbito quando da realização do procedimento. Não tivemos casos de infecção, trauma de órgãos maternos ou fetais e nem de trabalho de parto prematuro associado à transfusão intrauterina. Em todos os casos o sangramento no sítio da punção, quando da cordocentese, se resolveu espontaneamente em tempo inferior a 2 minutos.

O sangue utilizado foi do tipo $\mathrm{O}$ negativo com hematócrito de $80 \%$. Foi utilizado filtro de leucócitos na preparação do sangue devido ao fato de este processo apresentar melhor eficácia que a irradiação, além de que o sangue irradiado se torna mais espesso, dificultando sua infusão. $\mathrm{O}$ volume a ser transfundido seguiu a fórmula de Freda (idade gestacional em semanas - 20 multiplicado por $10 \mathrm{~mL}$ ), quando utilizada a técnica intraperitoneal. Nos casos de transfusão intravascular levamos em consideração a idade gestacional, o passado obstétrico, e o nivel da hemoglobina pré-transfusional para definição do volume a ser infundido ${ }^{3}$.

Em todos os casos o procedimento foi realizado com a paciente internada, que recebia alta após um período de observação de doze horas. Os fetos foram avaliados com ultra-sonografia, cardiotocografia e dopplerfluxometria arterial e venosa em tempo nunca superior a 6 horas antes e 6 a 12 horas após a realização das transfusões. O seguimento ambulatorial implicava, além da avaliação clínica da gestante, repetição do teste de Coombs indireto uma semana após o procedimento, repetição do painel de hemácias e a propedêutica fetal, que consistia de avaliação ultra-sonográfica, dopplerfluxometria venosa (veia cava inferior, ducto venoso e veia umbilical) e cardiotocografia semanal até o parto ou nova transfusão. 
Os critérios considerados como de piora do estado fetal foram a cardiotocografia não-reativa, alteração do Doppler venoso e sinais na ultrasonografia sugestivos ou compativeis com hidropisia (polidrâmnio, aumento da espessura placentária, medida do diâmetro bi-ventricular externo acima de 2 desvios-padrão, ascite, derrame pleural ou pericárdico).

A interrupção da gravidez foi programada para após a 34ㄹ semana gestacional na dependência dos niveis calculados da hematimetria fetal quando da realização de cordocentese e estimada a queda do hematócrito em relação ao peso fetal. A conduta obstétrica sempre foi individualizada levando-se em consideração o passado obstétrico, o nivel do teste de Coombs e os valores da espectrofotometria do líquido amniótico, assim como o resultado das provas de avaliação da vitalidade fetal, principalmente a cardiotocografia. Consideraram-se para efeito de avaliação dos resultados a mortalidade fetal e a neonatal precoce.

\section{Resultados}

Os resultados quanto à idade gestacional do início das transfusões são apresentados na Tabela 1. Destacamos a gravidade dos casos pela presença de $19,7 \%$ de fetos hidrópicos antes do início das transfusões.

Tabela 1 - Distribuição dos casos conforme a idade gestacional quando da transfusão intra-uterina inicial.

\begin{tabular}{lrr}
\hline $\begin{array}{l}\text { Idade gestacional } \\
\text { (semanas) }\end{array}$ & $\mathbf{n}$ & $\%$ \\
\hline$<$ de 20 & 4 & 6,6 \\
20 a 25 & 26 & 42,6 \\
25 a 30 & 25 & 41,0 \\
30 a 35 & 6 & 9,8 \\
Total & 61 & 100,0 \\
\hline
\end{tabular}

Em nossa casuística, aproximadamente em metade dos casos $(49,2 \%)$ a primeira transfusão ocorreu antes da 25a semana, sendo que em apenas 6 casos o primeiro procedimento ocorreu após a $30^{\mathrm{a}}$ semana.

A via intraperitoneal para a transfusão de sangue foi utilizada 81 vezes $(49,7 \%)$, a intravascular 78 vezes $(47,8 \%)$ e a combinação das duas em 4 oportunidades $(2,5 \%)$.

$O$ fato de que nos casos de transfusão intraperitoneal o sangue necessita ser absorvido no espaço subdiafragmático para entrar na circulação sangüínea acarretou que, nos casos em que se utilizou a via intraperitoneal, a interrupção da gestação sempre ocorreu 2 semanas após a última transfusão, para propiciar uma melhor absorção do sangue injetado.

Nos casos de fetos hidrópicos e que evoluíram de forma satisfatória, ocorreu a reversão do quadro após a segunda transfusão em dois casos e nos outros ocorreu apenas após a realização da terceira transfusão. Em todos estes casos a hemoglobina de cordão se encontrava abaixo de 8 $\mathrm{g} / \mathrm{dL}$ no sangue obtido antes da infusão. Estes casos tiveram sobrevida perinatal de apenas $42 \%$. O intervalo entre os procedimentos variou com a idade gestacional e com a gravidade dos casos, sendo que em 3 casos realizou-se novo procedimento uma semana após o primeiro procedimento e nos outros 8 casos repetiu-se o procedimento 2 semanas após. Um dos fetos hidrópicos evoluiu para o óbito durante o procedimento, sendo que este concepto apresentava hemoglobina no sangue de cordão de $3 \mathrm{~g} / \mathrm{dL}$, demonstrando a gravidade do quadro da doença.

Entre os fetos não-hidrópicos a sobrevida foi de $83,7 \%$ entre os hidrópicos de $41,7 \%$ (tab 2), mostrando com este resultado a importância do início precoce do tratamento intra-uterino. Outro aspecto importante a ser mostrado é o aumento do número de transfusões intravasculares, que representam nesta casuística a metade dos casos, com tendência de se tornarem ainda mais freqüentes na abordagem terapêutica dos fetos anêmicos.

Tabela 2 - Resultado perinatal dos fetos acompanhados conforme presença ou não da hidropisia fetal.

\begin{tabular}{lccrc}
\hline Fetos & \multicolumn{2}{c}{ Óbitos } & \multicolumn{2}{c}{ Vivos } \\
acompanhados & Fetais & Neonatais & n & $\%$ \\
\hline Não-hidrópicos & 5 & 3 & 41 & 83,7 \\
Hidrópicos & 4 & 3 & 5 & 41,7 \\
Total & 9 & 6 & 46 & \\
\hline
\end{tabular}

\section{Discussão}

Grande importância tem sido dada ao seguimento de fetos transfundidos ao longo dos primeiros anos de vida. Os estudos mais recentes mostram que o comprometimento do desenvolvimento neurológico é pequeno, embora não desprezível. Os casos mais graves de anemia que cursam com hidropisia apresentam maior risco de déficit neuromotor na infância ${ }^{1114}$. Este fato deve ser levado em consideração quando abordada uma gestante com risco de que o feto apresente um quadro de anemia, para que o tratamento seja reali- 
zado da forma mais adequada e o mais precocemente possivel.

Na Tabela 3 listamos algumas das principais revisões de casuísticas publicadas nos últimos anos, acrescentando, para comparação, os nossos resultados.

Tabela 3 - Resultados apresentados na literatura para a transfusão intra-uterina nas últimas três décadas.

\begin{tabular}{|c|c|c|c|c|}
\hline \multirow[t]{3}{*}{ Autor } & \multirow{3}{*}{$\begin{array}{c}\text { Casuística } \\
n^{0} \text { fetos }\end{array}$} & \multicolumn{2}{|c|}{ Sobrevida } & \multirow{2}{*}{$\begin{array}{l}\text { Sobrevida de } \\
\left.\right|^{*} \text { hidrópicos }\end{array}$} \\
\hline & & Via & perinatal* & \\
\hline & & & $\%$ & $\%$ \\
\hline Gregg e Hutchinson, $1969^{12}$ & 55 & $\mathbb{P}$ & 33 & - \\
\hline Hardyment et al., $1975^{13}$ & 57 & IP & 48 & - \\
\hline Rodeck et al., $1984^{19}$ & 13 & IV & 84 & - \\
\hline de Crespigny et al., $1985^{20}$ & 4 & IV & 75 & - \\
\hline Grannum et al., $1986^{21}$ & 20 & IV & 80 & - \\
\hline Berkowitz et al., $1988^{22}$ & 16 & IV & 76 & - \\
\hline Barss et al., $1988^{23}$ & 23 & IV & 85 & \\
\hline Poissonnier et al., $1989^{2}$ & 107 & IV & 86 & 78 \\
\hline \multirow[t]{2}{*}{ Harman et al., $1990^{18}$} & 44 & IP & 50 & 63 \\
\hline & 44 & IV & 83 & \\
\hline Doyle et al., $1993^{14}$ & 52 & IV & 73 & \\
\hline Hudon et al., $1998^{16}$ & 49 & $\mathrm{IV} / \mathrm{IP}$ & 81 & - \\
\hline Vitorello et al., $1998^{15}$ & 21 & IV & 80 & 62,5 \\
\hline Grab et al., $1999^{11}$ & 43 & IV & 81 & 63 \\
\hline CEMEFE-UFMG, 1999 & 61 & IV/IP & 84 & 42 \\
\hline
\end{tabular}

*Fetos hidrópicos e não-hidrópicos

IV- Intravascular

IP- Intraperitoneal

A gravidade dos casos acompanhados em nosso meio é bem demonstrada pelo fato de que aproximadamente $50 \%$ dos fetos necessitaram de tratamento com menos de 25 semanas.

Nossos fetos comprometidos receberam em média 2,7 transfusões, comparável ao citado por Harman et al. ${ }^{18}$. A idade média da interrupção da gravidez, em torno de 34,8 semanas, é a mesma verificada na maioria das séries citadas ${ }^{11}$.

Em relação à mortalidade perinatal encontrada em nossa casuística podemos verificar que situa-se próxima ao citado em revisões de outros centros com número de casos semelhantes aos nossos ${ }^{11,15,16}$. Os resultados obtidos em fetos hidrópicos ainda são muito preocupantes, mostrando a importância do diagnóstico precoce e tratamento rápido dos fetos comprometidos antes que os mesmos evoluam para a piora. O início precoce do tratamento fetal está associado a melhor prognóstico por duas razões. Primeiro, por corrigir a anemia precocemente, evitando o comprometimento da vitalidade fetal devido à insuficiência cardíaca. Em segundo lugar, as transfusões precoces inibem a eritropoese extramedular, o que também influencia a evolução perinatal ${ }^{11,18}$.

A técnica intravascular é, sem dúvida, a que oferece os melhores resultados tanto em nossos casos como naqueles citados pelos diversos auto$\operatorname{res}^{16,18}$.

Acreditamos que as transfusões intraperitoneais devam ser utilizadas em casos de dificuldade da punção vascular seja devido à placenta estar localizada na parede posterior, quando o concepto pela sua posição impede a punção do cordão umbilical, ou nos casos de fetos hidrópicos, pelo fato de estes conceptos absorverem de forma mais irregular o sangue infundido na cavidade peritoneal.

Finalizando, ressaltamos que os resultados da terapia do feto anêmico tem apresentado melhora ao longo das últimas décadas, passando de em torno de $30 \%$ na década de $60^{12}$, para próximo a $50 \%$ na década de $70^{13}$, estando a partir da década de 80 acima dos $80 \%{ }^{11,15,16,19}$. O ideal do ponto de vista de saúde pública é que a isoimunização materna possa ser reduzida ao mínimo pelo uso correto da imunoprofilaxia. Os casos inevitáveis, se existirem, devem ser reconhecidos precocemente pela detecção da anemia fetal e tratamento apropriado por meio da transfusão intravascular. Evitar a hidropisia fetal é conveniente para melhora do resultado perinatal e menor risco de comprometimento neurológico posterior.

\section{SUMMARY}

Purpose: to evaluate the intrauterine treatment of anemic fetuses that underwent intrauterine transfusions due to rhesus isoimmunization.

Methods: the authors studied sixty-one fetuses undergoing intrauterine transfusions by the intravascular, intraperitoneal or both routes. The hydropic fetuses (19.7\%) received only intravascular intrauterine transfusions. There was an overall number of 163 intrauterine transfusions with a mean of 2.7 procedures for each case. The indications for intrauterine transfusions were high values of bilirubin in amniotic fluid analyses by the Liley method or a hemoglobin concentration of cord blood below $10.0 \mathrm{~g} / \mathrm{mL}$.

Results: the overall perinatal survival rate was $46 \%$ for hydropic fetuses and $84 \%$ for the nonhydropic ones. There were no maternal side effects related to the procedures. Half of the intrauterine transfusions were performed by the intravascular route. The mean gestational age at the delivery was 34.8 weeks.

Conclusions: despite better perinatal results with intrauterine transfusions guided by ultrasound, especially using intravascular procedures, rhesus isoimmunization remains as an important cause of high rates of perinatal morbidity 
and mortality.

KEY WORDS: Isoimmunization. Intrauterine transfusion. Fetal therapy.

\section{Referências}

1. McSweeney E, Kirkham J, Vinall P, Flanagan P. An audit of anti-D sensitisation in Yorkshire. Br J Obstet Gynaecol 1998; 105:1091-4.

2. Poissonnier MH, Brossard Y, Demedeiros N, et al. Two hundred intrauterine exchange transfusions in severe blood incompatibilities. Am J Obstet Gynecol 1989; 161:709-13.

3. Cabral ACV, Filogônio CBB, Rezende CAL, Leite HV, Lima MIM, Corrêa MD. Avaliação da fórmula de cálculo da hemoglobina plasmática de fetos submetidos a transfusão intra-uterina. J Bras Ginecol 1988; 98:273-5.

4. Liley AW. Liquor amnii analysis in the management of the pregnancy complicated by rhesus sensitization. Am J Obstet Gynecol 1961; 82:1359-70.

5. Nicolaides KH, Sadovsky G, Cetin E. Fetal heart rate patterns in red blood cell isoimmunized pregnancies. Am J Obstet Gynecol 1989; 161:351-6.

6. Corrêa MD, Cabral ACV, Lima MIM. Cardiotocografia no seguimento de fetos submetidos à transfusão intra-uterina. J Bras Ginecol 1987; 97:381-4.

7. Mari G, Adrignolo A, Abuhamad AZ, et al. Diagnosis of fetal anemia with Doppler ultrasound in the pregnancy complicated by maternal blood group immunization. Ultrasound Obstet Gynecol 1995; 5:400-5

8. Liley AW. Intrauterine transfusion of foetus in haemolytic disease. Br Med J 1963; 2:1107-9.

9. Bang J, Bock JE, Trolle D. Ultrasound guided fetal intravenous transfusion for severe rhesus haemolytic disease. Br Med J (Clin Res Ed) 1982; 284:373-4.

10.Daffos F, Capella-Pavlovsky M, Forestier F. Fetal blood sampling via the umbilical cord using a needle guided by ultrasound. Report of 66 cases. Prenat Diagn 1983; 3:271-7.

11.Grab D, Paulus WE, Bommer A, Buck G, Terinde R. Treatment of fetal erythroblastosis by intravascular transfusions: outcome at 6 years. Obstet Gynecol 1999; 93:165-8.
12.Gregg GS, Hutchinson DL. Developmental characteristics of infants surviving fetal transfusion. JAMA 1969; 209:1059-62.

13.Hardyment AF, Salvador HS, Towell ME, Carpenter CW, Jan JE, Tingle AJ. Follow-up of intrauterine transfused surviving children. Am J Obstet Gynecol 1979; 133:235-41.

14.Doyle LW, Kelly EA, Richards AL, Ford GW, Callahan C. Sensorineural outcome at 2 years for survivors of erythroblastosis treated with fetal intravascular transfusions. Obstet Gynecol 1993; 81:931-5.

15.Vitorello DA, Parente LMM, Ramos RJ, Gonçalves LFA, Baumgarten CD, Saab Neto J. Transfusão intra-uterina em fetos afetados pela doença hemolitica perinatal grave: um estudo descritivo. Rev Bras Ginecol Obstet 1998; 20:137-44.

16.Hudon L, Moise KJ Jr, Hegemier SE, et al. Longterm neurodevelopmental outcome after intrauterine transfusion for the treatment of fetal hemolytic disease. Am J Obstet Gynecol 1998; 179:858-63.

17.Schumacher B, Moise KJ Jr. Fetal transfusion for red blood cell alloimmunization in pregnancy. Obstet Gynecol 1996; 88:137-50.

18.Harman CR, Bowman JM, Manning FA, Menticoglou SM. Intrauterine transfusion - intraperitoneal versus intravascular approach: a case-control comparison. Am J Obstet Gynecol 1990; 162:1053-9.

19.Rodeck CH, Nicolaides KH, Warsof SL, Fysh WJ, Gamsu HR, Kemp JR. The management of severe rhesus isoimmunization by fetoscopic intravascular transfusions. Am J Obstet Gynecol 1984; 150:769-74.

20.de Crespigny LC, Robinson HP, Quinn M, Doyle L, Ross A, Cauchi M. Ultrasound-guided fetal blood transfusion for severe rhesus isoimmunization. Obstet Gynecol 1985; 66:529-32.

21.Grannum PA, Copel JA, Plaxes SC, Scioscia AL, Hobbins JC. In utero exchange transfusion by direct intravascular injection in severe erythroblastosis fetalis. N Engl J Med 1986; 314:1431-4.

22.Berkowitz RL, Chitkara U, Wilkins IA, Lynch L, Plosker H, Bernstein HH. Intravascular monitoring and management of erythroblastosis fetalis. Am J Obstet Gynecol 1988; 158:783-95.

23.Barss VA, Benacerraf BR, Frigoletto FD, et al. Management of isoimmunized pregnancy by use of intravascular techniques. Am J Obstet Gynecol 1988; 159:932-7. 